\title{
Resistance to bacteriocin Lcn972 improves oxygen tolerance of Lactococcus lactis IPLA947 without compromising its performance as a dairy starter
}

\author{
María Jesús López-González , Ana Belén Campelo ${ }^{1}$, Antonia Picon ${ }^{2}$, Ana Rodríguez and Beatriz Martínez ${ }^{1 *}$ (D)
}

\begin{abstract}
Background: Lactococcus lactis is the main component of the mesophilic starters used in cheese manufacture. The success of milk fermentation relies on the viability and metabolic activity of the starter bacteria. Therefore, robust strains able to withstand the harsh conditions encountered during cheese manufacture and starter production are demanded. In this work, we have applied adaptive evolution under cell envelope stress imposed by the cell wall active bacteriocin Lcn972 to evolve strains with more robust phenotypes.

Results: Consecutive exposure of the starter strain L. lactis IPLA947 to Lcn972 yielded a stable mutant, L. lactis R5, with enhanced survival when challenged with hydrogen peroxide. L. lactis R5 exhibited faster growth rates in aerobic fermentations in broth and was able to acidify milk to a lower $\mathrm{pH}$ in aerated milk cultures. The improved behavior of L. lactis R5 in the presence of oxygen did not translate into a better performance in the presence of heme (i.e. respiration metabolism) or into higher survival during storage at cold temperatures or after freeze-drying compared to the wild type L. lactis IPLA947. L. lactis R5 retained the same milk acidification rate and no changes in the consumption of lactose and production of organic acids were noticed. However, the profile of volatile compounds revealed a significant increase in 3-hydroxy-2-butanone (acetoin) in curds manufactured with L. lactis R5.

Conclusions: Based on our results, L. lactis R5 can be proposed as a suitable dairy starter with improved survival under oxidative stress and enhanced metabolic traits. The results support the notion that adaptive evolution under cell envelope stress might be useful to generate strain diversity within industrial L. lactis strains.
\end{abstract}

Keywords: Dairy starter, Lactococcus lactis, Bacteriocin, Oxidative stress, Adaptive evolution, Lcn972

\section{Background}

Lactic acid bacteria (LAB) are the main components of dairy starters and are used to produce a wide variety of fermented products such as cheese, butter, fermented milk, yogurt, etc., which are highly appreciated by consumers. Among LAB, Lactococcus lactis is the main component of the mesophilic starters used in cheese manufacture. The main function of L. lactis is to produce $\mathrm{L}(+)$ lactic acid from lactose leading to the $\mathrm{pH}$ decrease required for milk clotting. The enzymatic pool

\footnotetext{
* Correspondence: bmf1@ipla.csic.es

${ }^{1}$ Dairy Safe group, Department of Technology and Biotechnology of Dairy Products, Instituto de Productos Lácteos de Asturias, IPLA-CSIC, Paseo Río Linares, s/n 33300 Villaviciosa, Asturias, Spain

Full list of author information is available at the end of the article
}

of L. lactis is further involved in the development of the flavor and texture of the final product [35]. For this reason, starter strains have been carefully selected according to their metabolic and technological properties along with the absence of undesirable traits (e.g. antibiotic resistance genes) [15]. Suitability for large scale production is also a major criterion in starter selection. A key step forward has been achieved by the introduction of a starter production strategy based on respiration (revised by [28]). Biomass yields and survival of Lactococcus and other LAB are greatly enhanced by aerobic growth in the presence of heme, i.e. under respiration metabolism where oxygen is reduced to water and protons are extruded $[14,30]$. Otherwise, the presence of oxygen

(c) The Author(s). 2018 Open Access This article is distributed under the terms of the Creative Commons Attribution 4.0 International License (http://creativecommons.org/licenses/by/4.0/), which permits unrestricted use, distribution, and 
originates reactive oxygen species that compromise $L$. lactis growth [7]. Indeed, several studies have confirmed that dissolved oxygen, as well as the positive redox potential of milk, can seriously compromise the acidification rate of $L$. lactis in milk [16, 22].

Considering that the success of milk fermentation relies on the viability and metabolic activity of $L$. lactis, continuous efforts have been made to understand the physiology behind robust phenotypes of industrially relevant microorganisms (reviewed by [27]). The knowledge gained on L. lactis stress physiology has been translated, mostly by recombinant DNA technology, into engineered strains with superior traits. However, legal constraints and the negative opinion of consumers towards genetically modified organisms (GMOs) pose a barrier for further marketing and create demand for alternative strategies $[12,20]$.

Adaptive evolution is becoming a popular non-GMO strategy to enhance starter fitness and develop evolved strains with enhanced technological traits. During adaptive evolution, bacterial populations are confronted with a particular stressor for several generations to select for mutations that enhance fitness under those prevailing conditions [37]. Adaptive evolution has been recently applied, for example, to improve $L$. lactis tolerance to high temperature [8] and acid tolerance in Lactobacillus casei and Leuconostoc mesenteroides [17, 38]. Interestingly, a common occurrence in adaptive evolution experiments is the selection of additional phenotypes not directly related to the applied stress, and which may likely arise due to mutations in global regulators, metabolic adaptations and/ or cross-protection phenomena $[8,27]$.

The aim of this work was to assess adaptive evolution under cell envelope stress as a means to enhance robustness of $L$. lactis. Cell envelope stress was applied by exposure to the anti-lactococcal bacteriocin Lcn972 that inhibits cell wall biosynthesis by specifically binding to the cell wall precursor lipid II, thereby triggering the cell envelope stress response in L. lactis [24, 25]. Previous work carried out with the laboratory strain $L$. lactis MG1614 (a derivative of the laboratory strain L. lactis MG1363) suggested that exposure to Lcn972 activated genes that contribute to survival to heat and acid stresses [31]. Moreover, Lcn972-resistant mutants of $L$. lactis MG1614 had a more densely packed peptidoglycan and increased resistance to nisin and lysozyme [32]. Based on these observations, we hypothesized that continuous exposure of L. lactis to Lcn972 might result in evolved strains with additional beneficial traits, besides becoming resistant to Lcn972. We have applied this strategy to the industrial strain L. lactis IPLA947 (CECT 5180), which is the main acidifying strain in a mixed-strain starter culture designed for the manufacture of the Afuega'l pitu cheese [3]. This cheese is an acid-coagulated cheese, made with pasteurized milk, in which coagulation relies heavily on the optimal activity of the added starter bacteria [10]. Because the initial levels of oxygen in milk may interfere with acidification by $L$. lactis, we have specifically studied if evolved strains could better withstand oxidative stress.

\section{Methods}

Bacterial strains and growth conditions

L. lactis IPLA947 [3] was incubated at $30{ }^{\circ} \mathrm{C}$ in $\mathrm{M} 17$ (Biokar Diagnostics, Beauvais, France) containing lactose $(0.5 \%)$ as a carbon source. Batch cultures were carried out in standing 12-ml tubes filled in with $10 \mathrm{ml}$ of M17 broth. Aerated cultures were grown in 50-ml Falcon tubes with culture volumes not exceeding $20 \mathrm{ml}$ and under shaking at $250 \mathrm{rpm}$. When required, hemin (Sigma-Aldrich, Alcobendas, Spain) was added to M17 at $0.01 \mathrm{mg} / \mathrm{ml}$ to activate respiration. Growth parameters were calculated from, at least, two independent cultures started by inoculating pre-warmed M17 with overnight cultures at an optical density at $600 \mathrm{~nm}\left(\mathrm{OD}_{600}\right)$ of 0.05 . Growth rates $(\mu)$ and generation times $(\mathrm{g})$ were calculated by linear regression of $\ln \left(\mathrm{OD}_{600}\right)$ versus time in the exponential phase and as $\ln (2) / \mu$, respectively. $\mathrm{OD}_{600}$ measurements were done in $1-\mathrm{cm}$ cuvettes in a Biophotometer (Eppendorf, Hamburg, Germany) or followed in a Benchmark Plus Microplate spectrophotometer (BioRad Laboratories, Hercules, CA, USA), as indicated. Colony forming units per milliliter $(\mathrm{CFU} / \mathrm{ml})$ were determined by plating serial decimal dilutions made in Ringer solution on M17 agar plates (Merck, Darmstadt, Germany).

\section{Adaptation to Lcn972 and susceptibility tests}

Lcn972 was purified and quantified as described elsewhere [25] and stocks $(320 \mu \mathrm{g} / \mathrm{ml}, 12,800 \mathrm{AU} / \mathrm{ml})$ were kept at $-20{ }^{\circ} \mathrm{C}$ in $50 \mathrm{mM}$ sodium phosphate buffer, $\mathrm{pH}$ 6.8. Adaptation was approached as previously described [32] with modifications. L. lactis IPLA947 was inoculated at $1 \%(\mathrm{v} / \mathrm{v})$ from an overnight culture and was sequentially cultivated at $30{ }^{\circ} \mathrm{C}$ for $16 \mathrm{~h}$ in M17 (2 ml) with doubling Lcn972 concentrations ranging from 20 to $1280 \mathrm{AU} / \mathrm{ml}$. Decimal dilutions from cultures grown with $1280 \mathrm{AU} / \mathrm{ml}$ were prepared in Ringer solution and plated on M17 agar to isolate single colonies. Selected colonies were consecutively grown in M17 for 100 generations in the absence of Lcn972. After colony purification, stabilized cultures were stored at $-80{ }^{\circ} \mathrm{C}$ in M17 broth supplemented with 10\% glycerol for further characterization. Minimal inhibitory concentrations (MICs) were determined during the stabilization step (i.e. growth without Lcn972) by the broth microdilution method [24]. To do that, overnight cultures were first adjusted to an $\mathrm{OD}_{600}$ of 0.5 and then further diluted 1:1000 to prepare a cell suspension with a concentration 
of $3 \times 10^{5} \mathrm{CFU} / \mathrm{ml}$, which was subsequently used to inoculate microtiter plates, containing a gradient of Lcn972 concentrations.

\section{Resistance to hydrogen peroxide}

Survival to oxidative stress was determined according to Dijkstra et al. [13] with some modifications. Briefly, overnight M17 cultures were resuspended in $50 \mathrm{mM}$ sodium phosphate buffer, $\mathrm{pH} 6.8$, and exposed to $5 \mathrm{mM}$ $\mathrm{H}_{2} \mathrm{O}_{2}$ for $2 \mathrm{~h}$ at $30{ }^{\circ} \mathrm{C}$ in a water bath. Decimal dilutions were plated on M17 agar plates. Untreated controls were handled equally but using sodium phosphate buffer instead of $\mathrm{H}_{2} \mathrm{O}_{2}$. Three independent cultures per strain were analyzed.

\section{Phase-contrast microscopy}

Aliquots of exponentially $(4 \mathrm{~h})$ and stationary $(24 \mathrm{~h})$ cultures were deposited on glass slides and observed with a DMi8 inverted microscope (Leica microsystems, Wetzlar, Germany). Cell length and width was measured in cells $(n=32)$ from four different fields per sample using the microscope built-in software LasX.

\section{Survival during cold storage}

Late exponentially growing cultures of L. lactis IPLA947 and R5 were used to inoculate $20 \mathrm{ml}$ of M17 at $\mathrm{OD}_{600}$ 0.05 . Subsequently, $10-\mathrm{ml}$ aliquots were transferred to 12-ml tubes for batch incubation at $30{ }^{\circ} \mathrm{C}$ or to $50-\mathrm{ml}$ tubes which were agitated at $250 \mathrm{rpm}$. Incubation proceeded for $14 \mathrm{~h}$ (early stationary phase) before storage at $4{ }^{\circ} \mathrm{C}$. Samples were taken at weekly intervals and viability $(\mathrm{CFU} / \mathrm{ml})$ was determined by plating decimal dilutions on M17 agar plates. Two independent cultures per strain were analyzed.

\section{Lyophilisation}

Cells from late exponentially growing batch cultures $(100 \mathrm{ml})$ of L. lactis IPLA947 and R5 were collected, washed with Ringer solution and resuspended in $10 \mathrm{ml}$ of $11 \%$ reconstituted Difco skim milk (Becton Dickinson, Franklin Lakes, NJ, USA). Samples were frozen at $-80{ }^{\circ} \mathrm{C}$ and lyophilized in a Virtis Freezemobile 12EL (VirTis, Gardiner, NY, USA) for $48 \mathrm{~h}$. Lyophilized powder was homogenized by vigorous shaking and stored in $50-\mathrm{ml}$ tubes at $4{ }^{\circ} \mathrm{C}$.

Milk acidification and detection of lactose and organic acids Milk cultures were carried out in commercially available homogenized UHT milk (CAPSA, Granda, Spain). Milk $(50 \mathrm{ml})$ was inoculated at $2 \%(v / \mathrm{v})$ with M17 overnight cultures, which were previously washed twice with Ringer solution, and then incubated at $21{ }^{\circ} \mathrm{C}$ for $19 \mathrm{~h}$. Acidification was monitored with a real-time pHmeter ORION $^{\mathrm{m}}$ Versa Star $^{\mathrm{mm}}$ (Thermo Scientific Inc., Waltham,
MA, USA), which recorded pH values every $15 \mathrm{~min}$. As descriptors of the acidification curves, we use maximum acidification rates $(\mathrm{Vm})$, defined as $\mathrm{pH}$ decrease $(\mathrm{mU})$ per min, the time interval at which the maximum acidification rate was maintained (Tm), as well as the time in $\min (\mathrm{Te})$ to reach $\mathrm{pH} 4.6$ [19]. Curves were performed in duplicate.

At the end of the incubation, samples $(1 \mathrm{ml})$ were homogenized with $5 \mathrm{ml}$ of $4.5 \mathrm{mM} \mathrm{H}_{2} \mathrm{SO}_{4}$ for $1 \mathrm{~h}$ at $37^{\circ} \mathrm{C}$ under continuous shaking, centrifuged $(16,000 \mathrm{x}$ g, $\left.15 \mathrm{~min}, 4{ }^{\circ} \mathrm{C}\right)$, and filtered $(0.22 \mu \mathrm{m})$. Lactose and organic acids were determined by HPLC using an ICSep ICE-ION-300 ion-exchange column (mobile phase $0.0085 \mathrm{~N} \mathrm{H}_{2} \mathrm{SO}_{4}$, operating temperature $65{ }^{\circ} \mathrm{C}$, flow rate $0.4 \mathrm{ml} \mathrm{min}{ }^{-1}$ ). A 996 Photodiode Array Detector (Waters) for the determination of organic acids (detection wavelength $210 \mathrm{~nm}$ ), and a Waters 410 differential refractometer for sugar determination (detection wavelength $280 \mathrm{~nm}$ ) were used. For quantification, regression equations $\left(R^{2} \geq 0.99\right)$ were calculated by using different concentrations of the corresponding standards.

\section{Detection of volatile compounds}

L. lactis IPLA947 and R5 were activated by two successive transfers in $10 \%$ reconstituted skim milk prior to inoculation at $1 \%(v / v)$ in fresh semi-skim pasteurized milk supplemented with $1 \mathrm{ml} \mathrm{l}^{-1}$ of a $100 \mathrm{~g} \mathrm{l}^{-1} \mathrm{CaCl}_{2}$ solution. They were grown in two independent experiments at $25{ }^{\circ} \mathrm{C}$ for $24 \mathrm{~h}$ in $100 \mathrm{ml}$ glass flasks. Cultures were transferred, with the help of a sterile spatula, to centrifuge tubes and centrifuged at $12857 \mathrm{x}$ g for $20 \mathrm{~min}$ in an Eppendorf 5810R centrifuge (Eppendorf, Hamburg, Germany). Pellets were wrapped in aluminium foil, vacuum packed in HT3050 plastic bags (Cryovac Sealed Air Corporation, Milano, Italy) and kept at $-35{ }^{\circ} \mathrm{C}$ until further analysis. Duplicate curd samples $(7 \mathrm{~g})$ were homogenized in a mechanical grinder with $14 \mathrm{~g}$ of anhydrous $\mathrm{Na}_{2} \mathrm{SO}_{4}$ (Merck, Darmstadt, Germany) and $25 \mu \mathrm{l}$ of an aqueous solution of $495 \mathrm{mg} \mathrm{l}^{-1}$ cyclohexanone (Sigma-Aldrich) added as internal standard. Volatile compounds were extracted by solid-phase microextraction (SPME) using a Divinylbenzene/Carboxen/Polydimethylsiloxane (DVB/CAR/PDMS) coated fibre (Supelco, Bellefonte, PA, USA). They were analyzed and identified by gas chromatography-mass spectrometry (GC-MS) (HP 6890-MSD HP 5973, Agilent, Palo Alto, CA, USA) as previously described [29].

\section{Statistical analyses}

Statistically significant differences were analyzed by Student t-test as implemented in Microsoft Excel 2010 (Microsoft Corporation). One-way analysis of variance was conducted with IBM SPSS Statistics (ver. 24.0.0.1) to compare cell length and width of IPLA947 and R5 
cells from aerobic and batch cultures. A $P$ value below 0.05 was considered statistically significant.

\section{Results and discussion}

We have explored exposure of a L. lactis dairy starter strain to the cell wall active bacteriocin Lcn972 as a means of obtaining evolved strains with robust phenotypes. The reasoning behind this was that resistance to Lcn972 in laboratory strains is accomplished through several mechanisms that, as a whole, contribute to enhanced survival under harsh conditions. Such mechanisms involved remodeling of the cell envelope towards a more densely packed peptidoglycan, the likely production of structural or surface polysaccharides and the activation of genes with protecting functions [1, 32, 33]. However, it was not possible to anticipate if similar results could be obtained with industrial or environmental L. lactis strains because they often differ phenotypically and genetically from laboratory strains [6, 18]. Therefore, as a proof of concept, we applied this strategy to the starter strain L. lactis IPLA947.

Selection of L. lactis IPLA947 derivatives resistant to Lcn972 The MIC of the bacteriocin Lcn972 for L. lactis IPLA947 was $20 \mathrm{AU} / \mathrm{ml}$ (Table 1). This value was similar to those described for other lactococcal strains isolated from commercial dairy starters and for the laboratory strain $L$. lactis MG1614 [23]. Adaptive evolution under cell envelope stress (CES) was conducted by a two-step process consisting of an adaptation step, which involved exposing the cultures to increasing amounts of Lcn972, and a subsequent stabilization step, in which bacterial cultures were grown for 100 generations in the absence of Lcn972. Adapted cultures were able to grow at bacteriocin concentrations of up to $1280 \mathrm{AU} / \mathrm{ml}$, which is 64 times the initial MIC and near the immunity levels (1600 AU/ml) provided by the Lcn972 immunity proteins in Lcn972 producers [2]. Higher concentrations inhibited growth and fully dense M17 cultures (i.e. $\left.\mathrm{OD}_{600}=2.0-2.5\right)$ could not be reached in $16 \mathrm{~h}$. The culture able to grow at $1280 \mathrm{AU} / \mathrm{ml}$ was serially diluted to isolate single colonies and twenty-two colonies were randomly selected. Their MICs ranged from > $160 \mathrm{AU} / \mathrm{ml}$ (9 clones), $160 \mathrm{AU} / \mathrm{ml}$ (12 clones) to $80 \mathrm{AU} / \mathrm{ml}$ (1 clone) (data not shown). Three resistant variants, with MICs over $160 \mathrm{AU} / \mathrm{ml}$ (R2A and R3A) and $80 \mathrm{AU} / \mathrm{ml}$ (R5A), were selected for further characterization. Partial sequencing of the $16 \mathrm{~S}$ rDNA and RAPD-PCR profiles confirmed the identity of these clones and excluded unintentional contamination (data not shown).

After adaptation, a single colony from each resistant variant was consecutively grown in M17 in the absence of selective pressure, to confirm that the higher Lcn972 MICs shown by these evolved clones was not transitory, i.e. as a result of the induction of the CES response orchestrated by the two-component system CesSR [25]. Contrary to our previous observation with the laboratory strain L. lactis MG1614 [32], the resistant phenotype of the selected clones was not lost during stabilization and they retained the same Lcn972 MIC recorded right after the adaptation step ("RnA" strains) (Table 1). From each stabilized culture of R2A, R3A and R5A, a single colony was stored and named R2, R3 and R5, respectively. To assess possible fitness costs associated to the Lcn972 resistant phenotype, growth of L. lactis IPLA947 and its Lcn972R derivatives in M17 was monitored in a microtiter plate reader at $30{ }^{\circ} \mathrm{C}$ (Table 1). All the resistant strains had similar growth rates and no significant differences were noted $(P>0.05)$ compared to the wildtype strain. Therefore, stable Lcn972 resistant mutants derived from this industrial strain could be isolated and the putative mutations accumulated during adaptive evolution did not seem to pose a physiological burden.

\section{Resistance to hydrogen peroxide}

To initially address if the Lcn972R mutants had become resistant to oxidative stress, their viability was measured after being challenged with $5 \mathrm{mM} \mathrm{H}_{2} \mathrm{O}_{2}$ (Fig. 1). Under these conditions, L. lactis R2 behaved as the wildtype strain, while L. lactis R3 was the most sensitive with a viability loss of $0.4 \mathrm{Log} \mathrm{CFU} / \mathrm{ml}$. In contrast, the viability loss of mutant L. lactis R5 was significantly reduced by

Table 1 Properties of L. lactis IPLA947 and its Lcn972-resistant derivatives R2, R3 and R5

\begin{tabular}{|c|c|c|c|c|c|c|c|}
\hline \multirow[t]{3}{*}{ L. lactis } & \multirow{3}{*}{$\begin{array}{l}\text { MIC } \\
\text { Lcn972 } \\
\text { (AU/ml) }\end{array}$} & \multicolumn{6}{|c|}{ Growth rate $(\mu)$ and generation time $(g)^{a}$} \\
\hline & & \multicolumn{2}{|l|}{ Batch } & \multicolumn{2}{|l|}{ Aeration $^{b}$} & \multicolumn{2}{|l|}{ Respiration } \\
\hline & & $\overline{\mu\left(h^{-1}\right)}$ & $g(h)$ & $\overline{\mu\left(h^{-1}\right)}$ & $g(h)$ & $\mu\left(h^{-1}\right)$ & $g(h)$ \\
\hline 947 (WT) & 20 & $0.64 \pm 0.10$ & $1.10 \pm 0.16$ & $0.38 \pm 0.02$ & $1.81 \pm 0.08$ & $0.86 \pm 0.01$ & $0.81 \pm 0.01$ \\
\hline R2 & $>160$ & $0.60 \pm 0.02$ & $1.16 \pm 0.05$ & $0.43 \pm 0.01^{*}$ & $1.62 \pm 0.04$ & ND & ND \\
\hline R3 & $>160$ & $0.63 \pm 0.02$ & $1.11 \pm 0.03$ & $0.44 \pm 0.01^{*}$ & $1.59 \pm 0.04$ & ND & ND \\
\hline R5 & 80 & $0.63 \pm 0.01$ & $1.10 \pm 0.02$ & $0.50 \pm 0.01^{* *}$ & $1.38 \pm 0.04^{*}$ & $0.84 \pm 0.01$ & $0.83 \pm 0.01$ \\
\hline
\end{tabular}

${ }^{\mathrm{a}}$ Two independent cultures were analysed. Results are expressed as means \pm standard deviation

${ }^{\text {b}}$ Significantly different from the wildtype (WT) L. lactis IPLA947. $\left(^{*}\right) P<0.05 ;\left(^{* *}\right) P<0.01$

ND: not determined 


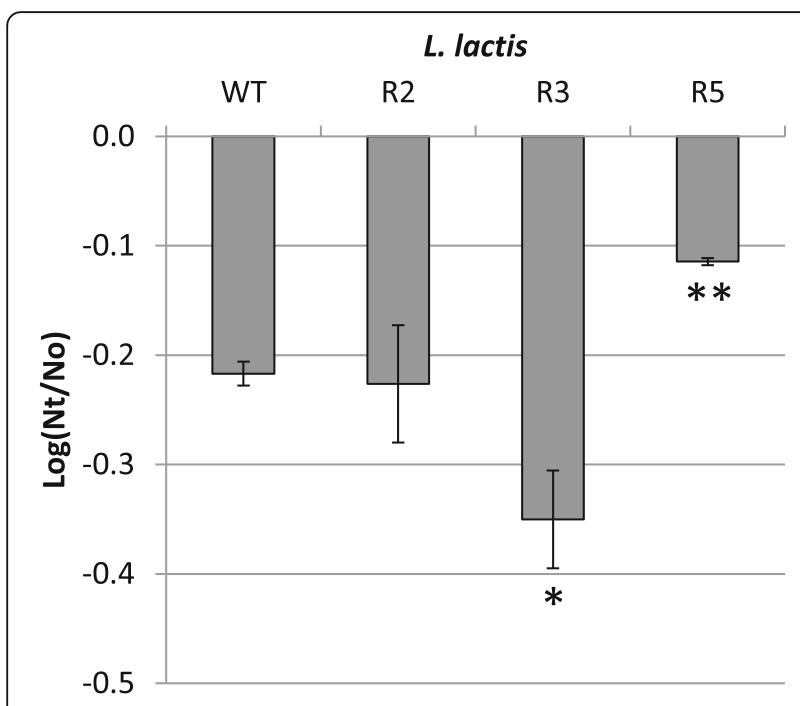

Fig. 1 Survival of L. lactis IPLA947 (WT) and its LCn972R derivatives L. lactis R2, R3 and R5 after exposure to $5 \mathrm{mM} \mathrm{H}_{2} \mathrm{O}_{2}$ for $2 \mathrm{~h}$ at $30^{\circ} \mathrm{C}$. $\mathrm{Nt}$ : CFU/ml after treatment; No: CFU/ml control. Results are the average of three independent cultures. Error bars are standard deviations. $\left(^{*}\right) P<0.05$; $\left(^{*}\right) P<0.01$, significantly different from L. lactis IPLA947

50\% $(P<0.01)$ compared to the wildtype strain, supporting the notion that $L$. lactis R5 coped with oxidative stress better after adaptive evolution under CES. These results anticipate the likely selection for different mutations within the population during adaptive evolution which is further supported by the different Lcn972 MICs (see Table 1).

\section{Growth of Lcn972 resistant derivatives in the presence of oxygen}

Resistance to oxidative stress was evaluated in aerated M17 cultures as well. Faster growth rates and reduced generation times (Table 1) were observed for all Lcn972R mutants over those of the wildtype strain. $L$. lactis $\mathrm{R} 2$ and $\mathrm{R} 3$ grew moderately faster $(P<0.05)$ than the wildtype strain but more slowly than L. lactis $\mathrm{R} 5$. In particular, the L. lactis R5 strain showed a 32\% improvement in growth rate.

Prompted by this result and the higher resistance of $L$. lactis $\mathrm{R} 5$ to $\mathrm{H}_{2} \mathrm{O}_{2}$, this mutant was further characterized. The performance of $L$. lactis $\mathrm{R} 5$ under respiration conditions (i.e. in the presence of hemin) was compared to that of the wildtype L. lactis IPLA947. In agreement with literature reports [28], both L. lactis IPLA947 and R5 grew faster under respiration conditions than in aerated cultures, almost doubling the growth rate (Table 1). However, the enhanced growth of the evolved L. lactis R5 in aerated cultures did not translate into a better performance under respiration as no significant differences in growth rate were observed between R5 and IPLA947
(Table 1). It has been reported that respiring lactococci are under lower oxidative stress due to the elimination of oxygen by the respiration metabolism [30]. Therefore, it is possible that the enhanced tolerance of L. lactis R5 to oxidative stress is masked under respiration, i.e. the mutations acquired during adaptive evolution may not represent an advantage under respiration conditions.

An intriguing observation during these growth experiments was that, while in batch cultures $L$. lactis IPLA947 and R5 strains reached similar maximum $\mathrm{OD}_{600}$ values, in aerated cultures the evolved $L$. lactis $\mathrm{R} 5$ consistently reached higher $\mathrm{OD}_{600}$ than the wildtype strain (Fig. 2a). However, this increment in $\mathrm{OD}_{600}$ did not translate into higher CFU counts which were 0.6 Log units lower than the CFU counts of L. lactis IPLA947 (Fig. 2b). Discrepancies between $\mathrm{OD}_{600}$ values and CFU counts might be explained by changes in cell morphology, by the presence of longer chains which would underestimate cell counts as one chain would yield a single colony, or by a viable but non-culturable state [36]. Hence, new aerated cultures of L. lactis IPLA947 and R5 strains were started and samples were taken for microscopy observation during exponential $(4 \mathrm{~h})$ and stationary $(24 \mathrm{~h})$ growth. Two main morphological features were observed by phase-contrast microscopy (Fig. 3). On the one hand, L. lactis R5 formed longer chains than the wildtype strain in aerated cultures during the exponential phase (Fig. 3a, b). Also, R5 cells were both longer and thicker than those of the wildtype strain (Fig. 3c, d), particularly after prolonged incubation times. These differences were not observed in batch cultures (Fig. 3). According to these results, the lower CFU counts of L. lactis R5 seem to be due to the formation of longer chains and the higher $\mathrm{OD}_{600}$ values night be related to the increase in cell size. To further confirm the enhanced growth of L. lactis $\mathrm{R} 5$ in the presence of oxygen, aerated cultures of L. lactis IPLA947 and R5 were carried out in milk and the $\mathrm{pH}$ decrease was measured as an indication of metabolic activity. Milk was inoculated in duplicate and incubated at $30{ }^{\circ} \mathrm{C}$ with vigorous shaking. During the first $8 \mathrm{~h}$, a similar trend was observed for both strains, but after $24 \mathrm{~h}$, L. lactis R5 reached a significantly $(p<0.05)$ lower $\mathrm{pH}$ value than the wildtype strain $(4.91 \pm 0.04$ vs $5.18 \pm 0.02)$ (Fig. 4). This result confirmed that the evolved L. lactis R5 performed better than the wildtype strain in the presence of oxygen not only in M17 broth but also in milk. This enhanced trait could have important technological consequences because the presence of oxygen in milk due to milk handling in the factory may slow down the acidification rate $[16,22]$.

\section{Survival during cold storage}

We sought to determine if the better performance of $L$. lactis R5 strain during aerobic growth compared to the 
a

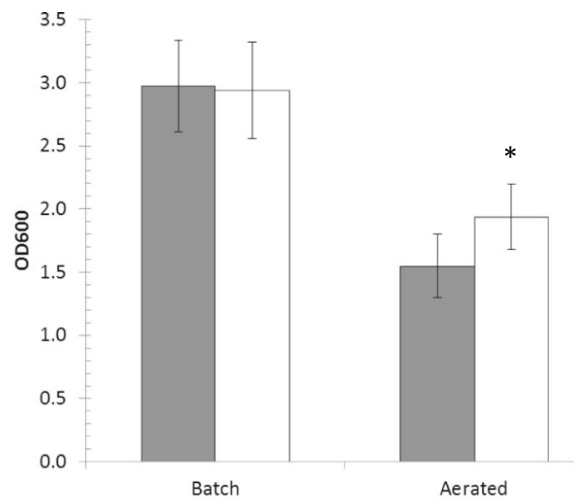

b

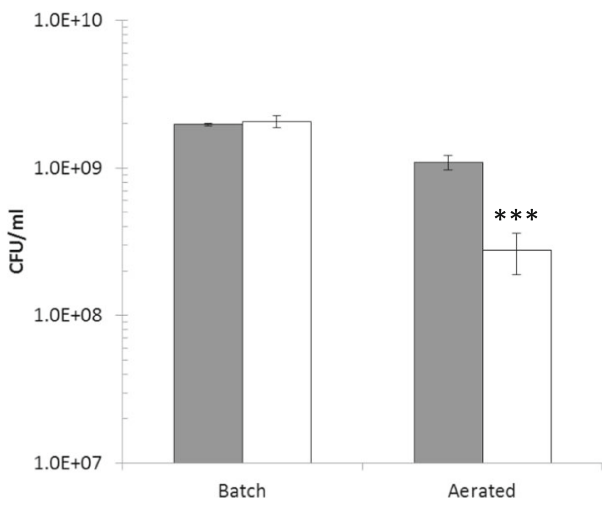

Fig. 2 Optical density $\left(\mathrm{OD}_{600}\right)$ (a) and CFU/ml (b) of batch and aerated cultures of L. lactis IPLA947 (grey bars) and the Lcn972R derivative L. lactis R5 (white bars) at the end of the exponential phase. Results are the average of two independent cultures. Error bars are standard deviations. $\left(^{*}\right) P<0.05 ;(* *) P<0.001$, significantly different from L. lactis IPLA947

wildtype strain also implied better viability upon storage at refrigeration temperatures. Thus, L. lactis IPLA947 and R5 strains were either incubated statically or shaken for $14 \mathrm{~h}$ prior to storage at $4{ }^{\circ} \mathrm{C}$ and samples were subsequently taken on a weekly basis to check their viability.
The initial bacterial counts remained constant for 3 weeks and decreased substantially after 5 weeks of storage (Fig. 5a). Survival of aerated cultures was roughly $3 \mathrm{Log}$ units higher compared to batch cultures $(P<0.05)$, which is in line with previous reports showing that fermentation

\section{a}

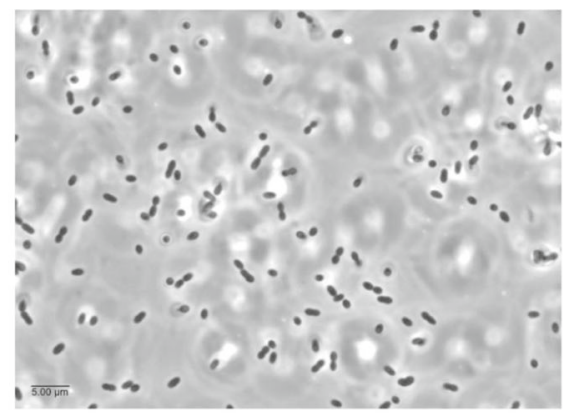

C

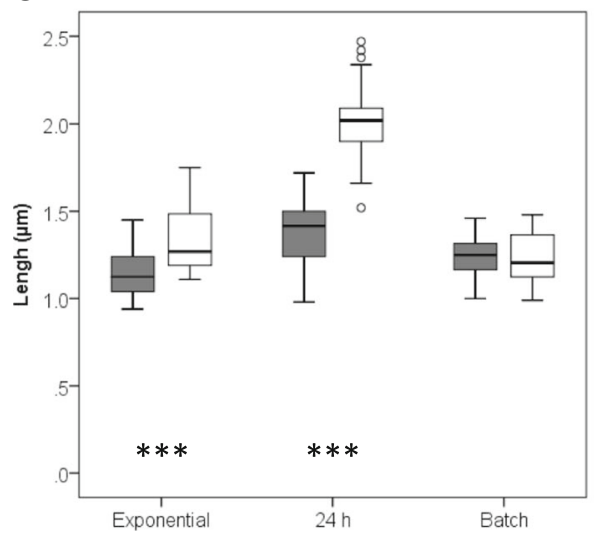

b

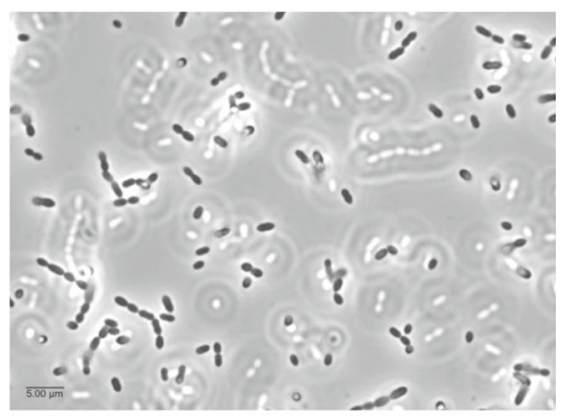

d

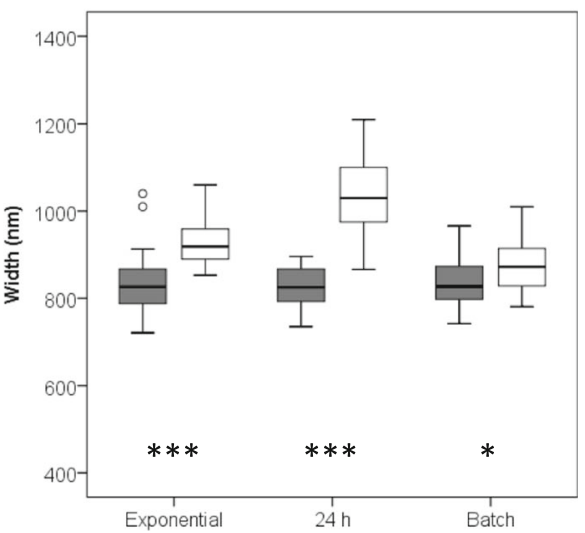

Fig. 3 Cell morphology of L. lactis IPLA947 and the LCn972R derivative L. lactis R5. Phase contrast microscopy of L. lactis IPLA 947 (a) and L. lactis R5 (b) sampled during growth in the presence of oxygen. Length (c) and width (d) of cells of L. lactis IPLA947 (grey) and L. lactis R5 (white) from exponentially and stationary $\left(24 \mathrm{~h}\right.$ ) aerated cultures and exponentially growing batch cultures. $\left(^{*}\right) P<0.05 ;\left(^{* * *}\right) P<0.001$, significantly different from L. lactis IPLA947 


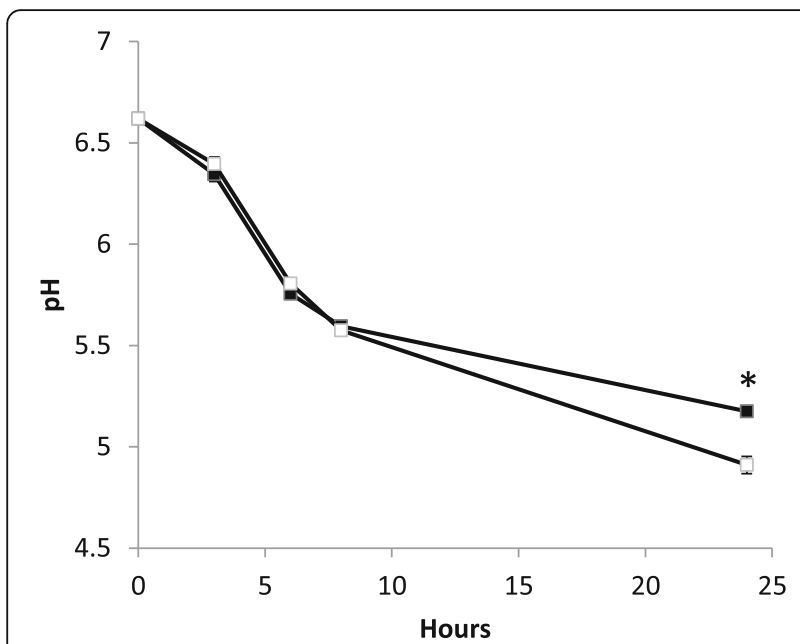

Fig. 4 Decrease of $\mathrm{pH}$ in L. lactis IPLA947 (black squares) and the Lcn972R mutant L. lactis R5 (white squares) in aerated cultures in milk. Results are the average of two independent cultures. Error bars are standard deviations. $\left(^{*}\right) P<0.05$, significantly different from L. lactis IPLA947

conditions may enhance $L$. lactis robustness and viability [13]. However, the R5 strain did not survive better than $L$. lactis IPLA947 $(P>0.05)$.

We also compared survival after freeze-drying and storage at $4{ }^{\circ} \mathrm{C}$ under air. Freeze-drying is a common method to preserve and deliver dairy starters, in which several factors including storage conditions are deemed relevant to maintain cell viability [4, 34]. For example, loss of membrane integrity due to oxidation of unsaturated fatty acids occurs during storage in the presence of oxygen, leading to reduced viability during storage [5]. As shown in Fig. 5b, the viability of L. lactis IPLA947 and R5 decreased during storage under air down to 3 Log units after 7 months but no differences were observed among them. Based on these results, the mutations acquired by L. lactis R5 seemed to protect it against exposure to oxygen during active growth but were not sufficient to guarantee better survival during storage.

\section{Performance of $L$. lactis R5 in milk fermentation}

Growth in milk of L. lactis R5 was followed to verify that no major technological traits were lost upon adaptation to Lcn972 and that the evolved L. lactis R5 strain would still be as suitable as a dairy starter as the parental strain L. lactis IPLA947. The acidification rate was tested at $21{ }^{\circ} \mathrm{C}$, the temperature at which Afuega'l Pitu cheese is coagulated [10]. As shown in Table 2, no significant differences $(P>0.05)$ were observed in any of the descriptors of the acidification curves. Similar maximum acidification rates were observed in both strains. The time to reach $\mathrm{pH} 4.6$, the isoelectric point of casein, was extended by an average of $45 \mathrm{~min}$ in L. lactis $\mathrm{R} 5$ but the data were not significantly different $(P>0.05)$ (Table 2$)$. The metabolic profile was also similar. After $19 \mathrm{~h}$ of incubation, residual lactose and production of the major organic acids (lactic, acetic and formic acids) were similar in both cultures (Table 2). Nonetheless, L. lactis R5 seemed to consume lactose more efficiently and showed a slightly higher mixed-acid metabolism compared to the wildtype strain, although these differences were not statistically significant $(P>0.05)$.

In a separate experiment, the volatile profile of curds made with L. lactis IPLA 947 and R5 strains was determined. After $24 \mathrm{~h}$ of incubation, eight volatile compounds (three acids, one alcohol and four ketones)
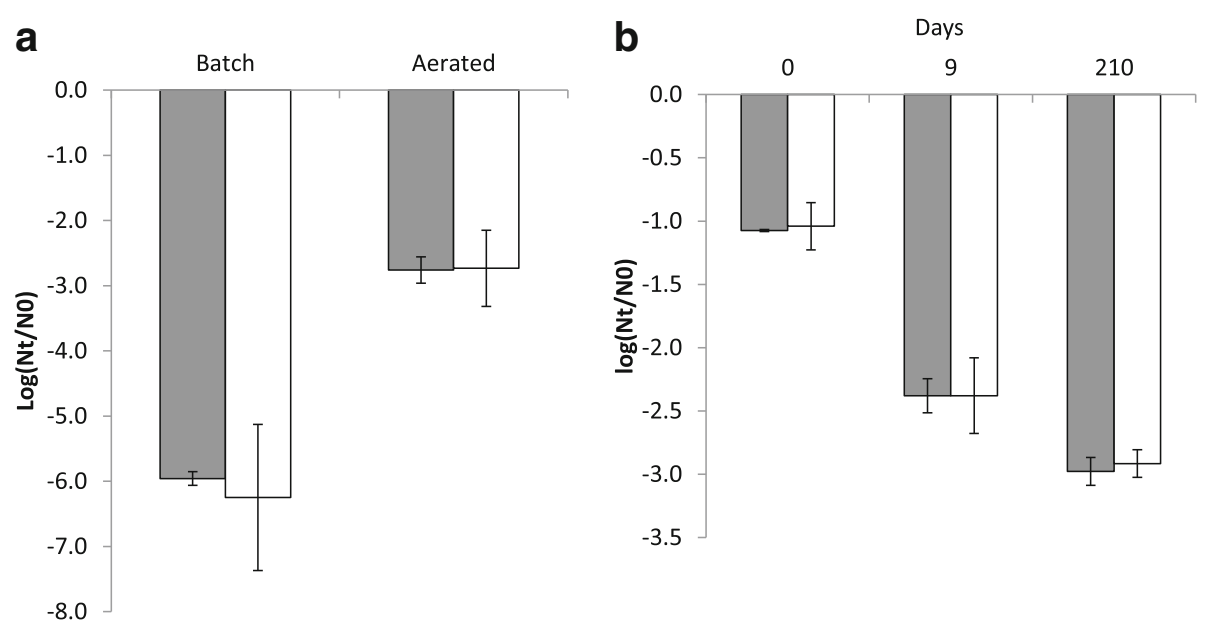

Fig. 5 Survival during cold storage of L. lactis IPLA947 (grey) and the Lcn972R derivative L. lactis R5 (white). (a) Viability of batch and aerated cultures in $\mathrm{M} 17$ broth stored for 5 weeks at $4{ }^{\circ} \mathrm{C}$. (b) Viability after freeze-drying and stored at $4{ }^{\circ} \mathrm{C}$ under air. Results are the average of two independent experiments. Error bars are standard deviations 
Table 2 Acidification and metabolites produced by L. lactis IPLA947 and the Lcn972 resistant mutant L. lactis R5 grown in milk

\begin{tabular}{|c|c|c|c|c|c|c|c|}
\hline \multirow[t]{2}{*}{ L. lactis strain } & \multicolumn{3}{|c|}{ Acidification parameters $^{\mathrm{a}}$} & \multirow[t]{2}{*}{ Residual lactose (mM) } & \multicolumn{3}{|c|}{ Organic acids (mM) } \\
\hline & $\overline{\mathrm{Vm} \text { (mpHUnits/min) }}$ & $\operatorname{Tm}(\min )$ & Te (min) & & Lactic & Acetic & Formic \\
\hline 947 (WT) & $3.27 \pm 0.04$ & 150 & $960 \pm 0$ & $103.8 \pm 6.5$ & $61.8 \pm 4.2$ & $0.68 \pm 0.07$ & $0.27 \pm 0.02$ \\
\hline R5 & $3.32 \pm 0.11$ & 150 & $1005 \pm 21$ & $91.5 \pm 4.9$ & $52.4 \pm 2.5$ & $1.01 \pm 0.02$ & $0.24 \pm 0.00$ \\
\hline
\end{tabular}

${ }^{a} V m$ maximum acidification rate, $\mathrm{Tm}$ time interval at which the maximum acidification rate was maintained, $T e$ time to reach pH 4.6. Results are expressed as means \pm standard deviation of two independent experiments

were detected. Levels of volatile compounds for both strains were not significantly $(P<0.05)$ different, with the only exception of 3-hydroxy-2-butanone (acetoin), whose levels were almost twofold higher in curds produced by L. lactis $\mathrm{R} 5$ than in those produced by L. lactis IPLA947 (Fig. 6). Acetoin is synthesized from pyruvate by the enzyme acetolactate synthase when the $\mathrm{NADH}$ pool is low and the activity of lactate and pyruvate dehydrogenases is not optimal [26]. The increased levels of acetoin in L. lactis R5 curd suggest activation of the acetolactate synthase pathway, diverting pyruvate metabolism towards acetoin production. This pathway has been shown to be activated to cope with the presence of oxygen in both M17 broth and milk cultures $[9,21]$. It seems plausible that, due to the stress imposed by Lcn972 during adaptive evolution, metabolic adaptations might have occurred as a strategy to produce additional energy and lower the stress level, as often described in the literature [27]. Therefore, mutations leading to the activation of the acetolactate synthase pathway might be behind the enhanced response of L. lactis $\mathrm{R} 5$ to oxidative stress. Moreover, acetoin, the reduction product of 2,3-butanodione (diacetyl), imparts a sour-milk aroma and together with diacetyl, which has buttery and vanilla notes, are key aroma components of many cheese varieties [11]. Hence, the incorporation of the evolved $L$. lactis R5 strain as part of the Afuega'l pitu cheese starter might improve cheese flavor. Nevertheless, Afuega'l pitu cheese pilot plant trials using pasteurized milk should be carried out to confirm the performance of the evolved strain under production conditions.

\section{Conclusions}

In this work, it has been shown that adaptive evolution of the industrial starter L. lactis IPLA947 under cell envelope stress may provide evolved strains with enhanced robustness phenotypes as exemplified by the higher tolerance to oxygen of L. lactis R5. Furthermore, adaptation to Lcn972 did not compromise essential technological parameters, such as milk acidification rate, and interesting metabolic changes were selected that might improve cheese flavour. Although it cannot be anticipated that every single $L$. lactis strain will evolve in a similar fashion as L. lactis IPLA947, our proposed strategy might be useful to generate strain diversity within industrial $L$. lactis strains, and could be adopted by the dairy industry in product development and market expansion.

The complexity of the stress-responsive regulatory networks that often overlap and provide cross-protection, together with additional metabolic adaptations, makes it difficult to predict and draw hypotheses on the putative mutations selected during adaptive evolution that are involved in resistance to the cell wall active Lcn972 and/or oxygen tolerance. Genome sequencing and transcriptional

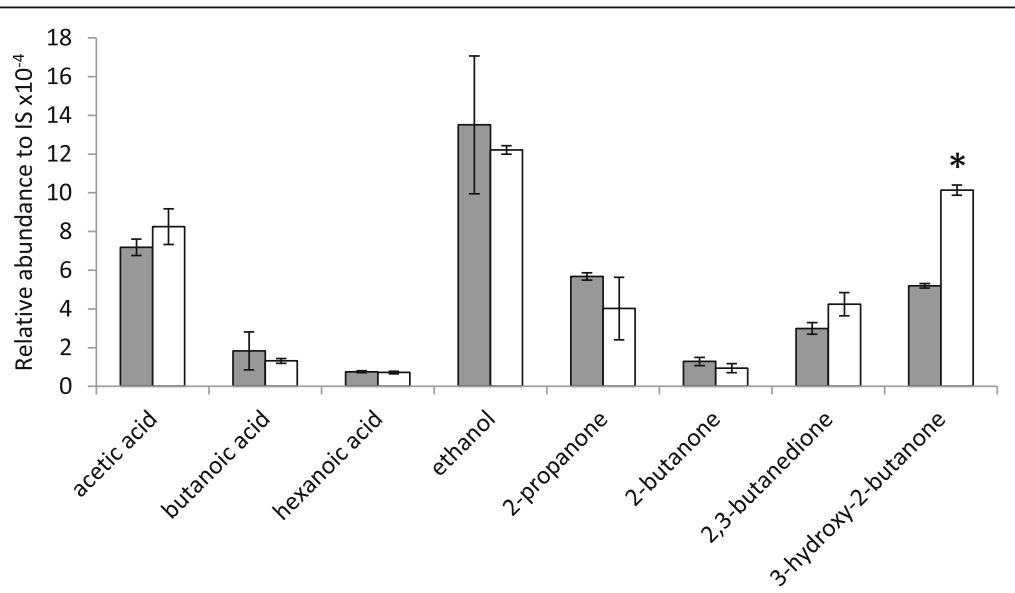

Fig. 6 Volatile compounds detected by GC-MS in milk cultures of L. lactis IPLA947 (grey bars) and the Lcn972R mutant L. lactis R5 (white bars). Results are the mean \pm standard deviation of two independent cultures. IS, internal standard $\left(^{*}\right) P<0.05$, significantly different from L. lactis IPLA947 
analyses will have to be approached in the near future to identify the molecular basis of the observed phenotypes and hopefully the results may contribute to a better understanding of the stress physiology of $L$. lactis.

\author{
Acknowledgements \\ The authors thank Olga López Pérez for her help with the GC-MS \\ analyses and Lucía Fernández for proofreading and English usage. We \\ acknowledge support of the publication fee by the CSIC Open Access \\ Publication Support Initiative through its Unit of Information Resources \\ for Research (URICI).
}

\section{Funding}

This research was supported by grant $\mathrm{BIO} 2013-46266-\mathrm{R}$ and $\mathrm{BIO2017-88147-}$ R (Ministerio de Economía y Competitividad, Spain). Activities of the DairySafe group at IPLA-CSIC were also funded by GRUPIN14-139 (FEDER funds and program of Science, Technology and Innovation 2013-2017, Principado de Asturias, Spain).

\section{Availability of data and materials}

All data generated or analyzed in this study are presented within this manuscript. All materials used in this study including raw data shall be available upon reasonable request.

\section{Authors' contributions}

$A R$ and $B M$ conceived and designed the study; MJLG, ABC, AP, and BM performed the experiments. All authors contributed to interpretation of the results and manuscript drafting. All authors read and approved the final manuscript.

\section{Ethics approval and consent to participate}

Not applicable.

\section{Consent for publication}

Not applicable.

\section{Competing interests}

The authors declare that they have no competing interests.

\section{Publisher's Note}

Springer Nature remains neutral with regard to jurisdictional claims in published maps and institutional affiliations.

\section{Author details}

'Dairy Safe group, Department of Technology and Biotechnology of Dairy Products, Instituto de Productos Lácteos de Asturias, IPLA-CSIC, Paseo Río Linares, s/n 33300 Villaviciosa, Asturias, Spain. ${ }^{2}$ Departamento de Tecnología de Alimentos, Instituto Nacional de Investigación y Tecnología Agraria y Alimentaria (INIA), Carretera de La Coruña Km 7.5, 28040 Madrid, Spain.

Received: 3 May 2018 Accepted: 10 July 2018

Published online: 20 July 2018

\section{References}

1. Campelo AB, Gaspar P, Roces C, Rodríguez A, Kok J, Kuipers OP, et al. The Lcn972 bacteriocin-encoding plasmid pBL1 impairs cellobiose metabolism in Lactococcus lactis. Applied Environmental Microbiology. 2011;77(21):7576-85. https://doi.org/10.1128/AEM.06107-11.

2. Campelo AB, Roces C, Mohedano ML, López P, Rodríguez A, Martínez B. A bacteriocin gene cluster able to enhance plasmid maintenance in Lactococcus lactis. Microb Cell Factories. 2014;13:77. https://doi.org/10.1186/ 1475-2859-13-77.

3. Cárcoba R, Delgado T, Rodríguez A. Comparative performance of a mixed strain starter in cow's milk, ewe's milk and mixtures of these milks. Eur Food Res Technol. 2000;211(2):141-6.

4. Carvalho AS, Silva J, Ho P, Teixeira P, Malcata FX, Gibbs P. Relevant factors for the preparation of freeze-dried lactic acid bacteria. Int Dairy J. 2004;14(10):835-47. https://doi.org/10.1016/j.idairyj.2004.02.001
5. Castro HP, Teixeira PM, Kirby R. Storage of lyophilized cultures of lactobacillus bulgaricus under different relative humidities and atmospheres. [journal article]. Appl Microbiol Biotechnol. 1995;44(1):172-6. https://doi.org/ 10.1007/bf00164498.

6. Cavanagh D, Casey A, Altermann E, Cotter PD, Fitzgerald GF, McAuliffe O. Evaluation of Lactococcus lactis isolates from nondairy sources with potential dairy applications reveals extensive phenotype-genotype disparity and implications for a revised species. Appl Environ Microbiol. 2015;81(12): 3961-72. https://doi.org/10.1128/AEM.04092-14.

7. Cesselin B, Derré-Bobillot A, Fernandez A, Lamberet G, Lechardeur D, Yamamoto $Y$, et al. Responses of lactic acid Bacteria to oxidative stress. In: Tsakalidou E, Papadimitriou K, editors. Stress responses of lactic acid Bacteria. Boston, MA: Springer US; 2011. p. 111-27.

8. Chen J, Shen J, Ingvar Hellgren L, Ruhdal Jensen P, Solem C. Adaptation of Lactococcus lactis to high growth temperature leads to a dramatic increase in acidification rate. Sci Rep. 2015;5:14199. https://doi.org/10.1038/srep14199.

9. Cretenet, M., Le Gall, G., Wegmann, U., Even, S., Shearman, C., Stentz, R., et al. (2014). Early adaptation to oxygen is key to the industrially important traits of Lactococcus lactis ssp. cremoris during milk fermentation. BMC Genomics, 15, 1054, https://doi.org/10.1186/1471-2164-15-1054.

10. Cuesta P, Fernández-García E, De Llano DG, Montilla A, Rodríquez A. Evolution of the microbiological and biochemical characteristics of Afuega'l Pitu cheese during ripening. J Dairy Sci. 1996;79(10):1693-8.

11. Curioni PMG, Bosset JO. Key odorants in various cheese types as determined by gas chromatography-olfactometry. Int Dairy J. 2002;12:959-84.

12. Derkx PM, Janzen T, Sørensen Kl, Christensen JE, Stuer-Lauridsen B, Johansen $\mathrm{E}$. The art of strain improvement of industrial lactic acid bacteria without the use of recombinant DNA technology. Microb Cell Factories. 2014;13(Suppl 1):S5. https://doi.org/10.1186/1475-2859-13-S1-S5.

13. Dijkstra AR, Alkema W, Starrenburg MJ, Hugenholtz J, van Hijum SA, Bron PA. Fermentation-induced variation in heat and oxidative stress phenotypes of Lactococcus lactis MG1363 reveals transcriptome signatures for robustness. Microb Cell Factories. 2014;13:148. https://doi.org/10.1186/ s12934-014-0148-6.

14. Duwat P, Sourice S, Cesselin B, Lamberet G, Vido K, Gaudu P, et al. Respiration capacity of the fermenting bacterium Lactococcus lactis and its positive effects on growth and survival. J Bacteriol. 2001;183(15):4509-16. https://doi.org/10.1128/JB.183.15.4509-4516.2001

15. Hansen EB. Commercial bacterial starter cultures for fermented foods of the future. Int J Food Microbiol. 2002;78(1-2):119-31. https://doi.org/10.1016/ S0168-1605(02)00238-6.

16. Jeanson S, Hilgert N, Coquillard M-O, Seukpanya C, Faiveley M, Neveu P, et al. Milk acidification by Lactococcus lactis is improved by decreasing the level of dissolved oxygen rather than decreasing redox potential in the milk prior to inoculation. Int J Food Microbiol. 2009;131(1):75-81. https://doi.org/ 10.1016/j.jifoodmicro.2008.09.020

17. Ju SY, Kim JH, Lee PC. Long-term adaptive evolution of Leuconostoc mesenteroides for enhancement of lactic acid tolerance and production. Biotechnology for Biofuels. 2016;9:240

18. Kelleher P, Murphy J, Mahony J, van Sinderen D. Next-generation sequencing as an approach to dairy starter selection. Dairy Sci Technol. 2015;95(5):545-68. https://doi.org/10.1007/s13594-015-0227-4

19. Kristo E, Biliaderis CG, Tzanetakis N. Modelling of the acidification process and rheological properties of milk fermented with a yogurt starter culture using response surface methodology. Food Chem. 2003;83(3):437-46. https://doi.org/10.1016/S0308-8146(03)00126-2

20. Kuipers OP. Back to nature: a revival of natural strain improvement methodologies. Microb Biotechnol. 2015;8(1):17-8. https://doi.org/10.1111/ 1751-7915.12232.

21. Larsen N, Moslehi-Jenabian S, Werner BB, Jensen ML, Garrigues C, Vogensen FK, et al. Transcriptome analysis of Lactococcus lactis subsp. lactis during milk acidification as affected by dissolved oxygen and the redox potential. Int J Food Microbiol. 2016;226:5-12. https://doi.org/10.1016/j.ijfoodmicro. 2016.03.002.

22. Larsen N, Werner BB, Vogensen FK, Jespersen L. Effect of dissolved oxygen on redox potential and milk acidification by lactic acid bacteria isolated from a DL-starter culture. J Dairy Sci. 2015;98(3):1640-51. https://doi.org/10. 3168/jds.2014-8971.

23. Madera C, García P, Rodríguez A, Suárez JE, Martínez B. Prophage induction in Lactococcus lactis by the bacteriocin Lactococcin 972. Int J Food Microbiol. 2009;129(1):99-102. https://doi.org/10.1016/j.iffoodmicro.2008.11.004. 
24. Martínez B, Böttiger T, Schneider T, Rodríguez A, Sahl HG, Wiedemann I. Specific interaction of the unmodified bacteriocin Lactococcin 972 with the cell wall precursor lipid II. Appl Environ Microbiol. 2008;74(15):4666-70. https://doi.org/10.1128/AEM.00092-08.

25. Martínez B, Zomer AL, Rodríguez A, Kok J, Kuipers OP. Cell envelope stress induced by the bacteriocin Lcn972 is sensed by the lactococcal twocomponent system CesSR. Mol Microbiol. 2007;64(2):473-86. https://doi.org/ 10.1111/j.1365-2958.2007.05668.x.

26. Neves AR, Pool WA, Kok J, Kuipers OP, Santos H. Overview on sugar metabolism and its control in Lactococcus lactis - the input from in vivo NMR. FEMS Microbiol Rev. 2005;29(3):531-54. https://doi.org/10.1016/j. femsre.2005.04.005.

27. Papadimitriou K, Alegria A, Bron PA, de Angelis M, Gobbetti M, Kleerebezem $\mathrm{M}$, et al. Stress physiology of lactic acid Bacteria. Microbiol Mol Biol Rev. 2016:80(3):837-90. https://doi.org/10.1128/MMBR.00076-15.

28. Pedersen MB, Gaudu P, Lechardeur D, Petit MA, Gruss A. Aerobic respiration metabolism in lactic acid bacteria and uses in biotechnology. Annu Rev Food Sci Technol. 2012;3:37-58. https://doi.org/10.1146/annurev-food022811-101255.

29. Picon A, Fernández-García E, Gaya P, Nuñez M. Modification of the volatile compound profile of cheese by a Lactococcus lactis strain expressing a mutant oligopeptide binding protein. J Dairy Res. 2008;75:30-6.

30. Rezaïki L, Cesselin B, Yamamoto Y, Vido K, van West E, Gaudu P, et al. Respiration metabolism reduces oxidative and acid stress to improve longterm survival of Lactococcus lactis. Mol Microbiol. 2004;53(5):1331-42. https://doi.org/10.1111/j.1365-2958.2004.04217.x.

31. Roces C, Campelo AB, Veiga P, Pinto JPC, Rodríguez A, Martínez B Contribution of the CesR-regulated genes IImg0169 and IImg2164-2163 to Lactococcus lactis fitness. Int J Food Microbiol. 2009;133(3):279-85. https://doi.org/10.1016/j.ijfoodmicro.2009.06.002.

32. Roces C, Courtin P, Kulakauskas S, Rodríguez A, Chapot-Chartier MP, Martínez B. Isolation of Lactococcus lactis mutants simultaneously resistant to the cell wall-active bacteriocin Lcn972, lysozyme, nisin and bacteriophage c2. Appl Environ Microbiol. 2012a;78(12):4157-63. https://doi.org/10.1128/AEM.00795-12.

33. Roces C, Pérez V, Campelo AB, Blanco D, Kok J, Kuipers OP, et al. The putative lactococcal extracytoplasmic function anti-sigma factor IImg2447 determines resistance to the cell wall-active bacteriocin Icn972. Antimicrob Agents Chemother. 2012b;56(11):5520-7. https://doi.org/10.1128/AAC.01206-12.

34. Santivarangkna C, Kulozik U, Foerst P. Alternative drying processes for the industrial preservation of lactic acid starter cultures. Biotechnol Prog. 2007;23(2):302-15. https://doi.org/10.1021/bp060268f.

35. Smid EJ, Kleerebezem M. Production of aroma compounds in lactic fermentations. Annu Rev Food Sci Technol. 2014;5:313-26. https://doi.org/ 10.1146/annurev-food-030713-092339.

36. Stevenson K, McVey AF, Clark IB, Swain PS, Pilizota T. General calibration of microbial growth in microplate readers. Sci Rep. 2016;6:38828. https://doi. org/10.1038/srep38828.

37. Zelder $\mathrm{O}$, Hauer $\mathrm{B}$. Environmentally directed mutations and their impact on industrial biotransformation and fermentation processes. Curr Opin Microbiol. 2000;3(3):248-51.

38. Zhang J, Wu C, Du G, Chen J. Enhanced acid tolerance in Lactobacillus casei by adaptive evolution and compared stress response during acid stress. Biotechnol Bioprocess Eng. 2012;17:283-98.

Ready to submit your research? Choose BMC and benefit from:

- fast, convenient online submission

- thorough peer review by experienced researchers in your field

- rapid publication on acceptance

- support for research data, including large and complex data types

- gold Open Access which fosters wider collaboration and increased citations

- maximum visibility for your research: over $100 \mathrm{M}$ website views per year

At BMC, research is always in progress.

Learn more biomedcentral.com/submissions 